\title{
Cutsets in infinite graphs
}

\author{
BY ÁDÁM TimÁR
}

\begin{abstract}
We answer three questions posed in a paper by Babson and Benjamini. They introduced a parameter $C_{G}$ for Cayley graphs $G$ that has significant application to percolation. For a minimal cutset of $G$ and a partition of this cutset into two classes, take the minimal distance between the two classes. The supremum of this number over all minimal cutsets and all partitions is $C_{G}$. We show that if it is finite for some Cayley graph of the group then it is finite for any (finitely generated) Cayley graph. Having an exponential bound for the number of minimal cutsets of size $n$ separating $o$ from infinity also turns out to be independent of the Cayley graph chosen. We show a 1-ended example (the lamplighter group), where $C_{G}$ is infinite. Finally, we give a new proof for a question of de la Harpe, proving that the number of $n$-element cutsets separating $o$ from infinity is finite unless $G$ is a finite extension of $\mathbb{Z}$
\end{abstract}

\section{$\S 1$. Introduction.}

In their paper $[\mathrm{BB}]$, Babson and Benjamini introduce a parameter $C_{G}$ for an infinite Cayley graph $G$ in the following way. Let $G_{+}$be the set of vertices in $G$ together with the set of ends of $G$. Given a subset $Y$ in $V(G) \cup E(G)$, let

$$
C(Y)=\sup _{Y_{1} \cup Y_{2}=Y}\left\{\operatorname{dist}\left(Y_{1}, Y_{2}\right)\right\}
$$

If $C(Y) \leq t$, then $Y$ is said to be $t$-close. Let

$$
C_{G}=\sup C(\Pi)
$$

where $\Pi$ ranges over all minimal cutsets between points $x, y \in G_{+}$.

For example, it is easy to check that for the square-grid we have $C_{\mathbb{Z}^{2}}=2$, while $C_{G}=3$ for the hexagonal grid $G$. The so called lamplighter group will turn out to be such that its $C_{G}$ is infinity (see Section 4 ).

2000 Mathematics Subject Classification. Primary 05C25. Secondary 20F65.

Key words and phrases. Cutsets, quasi-isometry.

Research partially supported by NSF Grant DMS-0231224 and Hungarian National Foundation for Scientific Research Grants TO34475. 
An exponential bound on the number of minimal cutsets of size $n$ provides one with a nontrivial upper bound on the percolation critical probability $p_{c}$ of $G$. Moreover, if $C_{G}$ is bounded for some one-ended graph $G$ then the critical probability $p_{u}$ for having a unique infinite cluster is smaller than 1 , as shown in $[\mathrm{BB}]$. There it is also shown that for finitely presented groups $G$ (i.e., groups with finitely many defining relators) $C_{G}$ is finite. We shall give a shorter and elementary proof for that in Section 5. This established positively Question 3 in [BS] for finitely presented groups, showing that a finitely group with one end has $p_{u}<1$. The original question, whether $p_{u}$ is smaller than 1 in finitely generated groups with one end is still open, so it is natural to ask whether $C_{G}$ is finite for any Cayley graph $G$.

At the end of $[\mathrm{BB}]$ there are three questions:

Question 1: Does having some exponential bound on the number of minimal cutsets of size $n$ in a Cayley graph depend only on the group and not on the choice of generators?

Question 2: Does the property " $C_{G}$ is finite" for a Cayley graph depend only on the group and not on the choice of generators?

Question 3: Are there finitely generated groups with one end so that $C_{G}$ is not finite?

In Section 2 and Section 3 we give positive answers to the first and the second question of $[\mathrm{BB}]$ respectively. In both cases we prove the invariance not only for different Cayley graphs of the same group, but for any two graphs that have bounded degrees and that are quasi-isometric under a bijection. Similar, but lengthier, arguments would show that the same conclusions hold for any two quasi-isometric bounded degree graphs.

In Section 4 we show that the lamplighter group is an example of a group with one end and infinite $C_{G}$, hence answering Question 3 negatively.

Babson and Benjamini conjecture that for any Cayley graph the number of minimal cutsets separating a fixed vertex $o$ from infinity is exponentially bounded in the size of the cutset. They prove it for finitely presented groups. Problem VI.19 from [dlH] is weaker: "In a finitely-generated group which is not almost cyclic, does the size of spheres tend to infinity?" (A group is almost cyclic if it is a finite extension of $\mathbb{Z}$.) We give a short proof in Section 6 for that the sizes of any sequence of (distinct) cutsets tend to infinity. This have already been shown by Anna Erschler (see the appendix of the second edition of $[\mathrm{dlH}]$ ), using a result of Coulhon and Saloff-Coste ([CS]).

For any set $X$ of vertices of a graph we denote by $N_{n}(X)$ the $n$-neighborhood of $X$, that is, the set of points at distance $\leq n$ from $X$ in the graph. Note that although $N_{n}(X)$ will be used for different graphs, it is the set $X$ that indicates which graph is understood.

In what follows, for simpler phrasing, cutset will always mean a set of edges whose deletion disconnects the graph. Everything remains valid if we use cutsets consisting of 
vertices.

We do not always distinguish between vertex sets and subgraphs induced by them.

A bijective quasi-isometry (or bi-Lipschitz map) with constant $m$ between the graphs $G=(V, E)$ and $G^{\prime}=\left(V^{\prime}, E^{\prime}\right)$, is a bijection $f$ from $V(G)$ to $V\left(G^{\prime}\right)$ such that the following holds:

For any $x, y \in V(G)$,

$$
1 / m \cdot \operatorname{dist}_{G}(x, y) \leq \operatorname{dist}_{G^{\prime}}(f(x), f(y)) \leq m \cdot \operatorname{dist}_{G}(x, y)
$$

If there is such a map between $G$ and $G^{\prime}$, we shall say that they are bijectively quasiisometric. Note that different Cayley graphs of the same group are always bijectively quasi-isometric.

Given a subset $X$ of $G, \partial X$ will denote its external boundary, that is, the set of vertices not in $X$ but adjacent to some vertex of $X$. We use $\partial_{i} X$ for the inner boundary.

\section{$\S 2$. The invariance of exponential bound.}

Given a graph $G$ and $o \in V(G)$, we say that there is an exponential bound for the number of minimal cutsets separating $o$ from infinity if there is a constant $\alpha$ such that the number of minimal cutsets of size $n$ separating $o$ from infinity is at most $\alpha^{n}$.

In this section we prove that having an exponential bound for the number of minimal cutsets in $o$ is invariant under bijective quasi-isometries.

We shall need the following lemma, which is basically Lemma 6 in [BB]; a stronger bound is given in $[\mathrm{Ke}]$.

Lemma 2.1. Let $G$ be a graph of degrees at most $d$ and o a fixed vertex of it. The number of subsets of vertices of size $n$ that contain o and induce a connected subgraph in $G$ is at most $d^{2 n}$.

Proof. Choose a spanning tree in an induced subgraph as above and define a "depth-first walk" in it: a walk that visits every vertex and goes through each edge at most twice. The walk determines the set of vertices, it has length $\leq 2 n$, and in each step there are at most $d$ ways to continue such a walk.

THEOREM 2.2. Let $\iota$ be a bijective quasi-isometry from a graph $G$ to $G^{\prime}$ with constant $m$. Suppose that the degrees in $G$ and $G^{\prime}$ are bounded by $d$. Let o be some fixed vertex of $G$. Then there is an exponential bound for the number of minimal cutsets in Geparating o 
from infinity if and only if there is an exponential bound in $G^{\prime}$ for the number of cutsets separating $\iota(o)$ from infinity.

Proof. Denote by $C_{n}$ and $C_{n}^{\prime}$ the set of minimal cutsets of size $n$ separating $o$ and $o^{\prime}:=\iota(o)$ from infinity in $G$ and $G^{\prime}$ respectively. Let $K_{n}\left(K_{n}^{\prime}\right)$ be the set of connected subgraphs that arise as the connected component containing $o\left(o^{\prime}\right)$ after removing an element of $C_{n}$ $\left(C_{n}^{\prime}\right)$ from $G\left(G^{\prime}\right)$. We shall define a map $\phi$ from $K_{n}$ to $H_{n}:=\bigcup_{j=1}^{n d^{2 m}} K_{j}^{\prime}$. The map $\phi$ will have the property that for every $\chi \in H_{n}$ the set $\left\{\kappa \in K_{n}: \phi(\kappa)=\chi\right\}$ has at most $c^{n}$ elements for some constant $c$ independent of $n$. The existence of $\phi$ shows that if $\left|K_{n}^{\prime}\right|$ (and hence $\left|H_{n}\right|$ ) is at most exponential, then $\left|K_{n}\right| \leq\left|H_{n}\right| c^{n}$ is also at most exponential. Hence the theorem will follow.

So, let $\kappa$ be an element in $K_{n}$. Define $\phi(\kappa)$ to be $N_{m}(\iota(\kappa))$. If $x, y \in V(\kappa)$ are adjacent in $G$ then there is a path of length $\leq m$ in $G^{\prime}$ between $\iota(x)$ and $\iota(y)$. So the vertices of $\iota(\kappa)$ are in one component of $\phi(\kappa)$, and since any other vertex in $\phi(\kappa)$ is in the same component of $\phi(\kappa)$ as some vertex of $\iota(\kappa)$, we see that $\phi(\kappa)$ is connected. Now $\phi(\kappa)$ contains $o^{\prime}$. Moreover, for any (external) boundary vertex $y$ of $\iota(\kappa)$ in $G^{\prime}, \iota^{-1}(y)$ is at distance at most $m$ from a boundary vertex of $\kappa$ in $G$. The set of vertices that are at distance $\leq m$ from $\partial \kappa$ in $G$ have cardinality $\leq d^{m}|\partial \kappa|$, so we get that $|\partial \iota(\kappa)| \leq d^{m}|\partial \kappa|$. Since $\phi(\kappa)$ is the $m$-neighborhood of $\iota(\kappa)$ in $G^{\prime}$, the boundary of $\phi(\kappa)$ is in the $m$-neighborhood of $\partial \iota(\kappa)$ in $G^{\prime}$, so $|\partial \phi(\kappa)| \leq d^{m}|\partial \iota(\kappa)|$. We get $|\partial \phi(\kappa)| \leq d^{2 m}|\partial \kappa|$ from these two inequalities. Hence $\phi(\kappa)$ is indeed in $H_{n}$.

What remains to be shown is that $\left|\left\{\kappa \in K_{n}: \phi(\kappa)=\chi\right\}\right| \leq c^{n}$ for any $\chi \in H_{n}$.

Fix $\chi \in H_{n}$ and let $\tau:=\iota^{-1}(\chi)$. If $\phi(\kappa)=\chi$ for some $\kappa \in K_{n}$ then $\kappa \subset \tau$. Furthermore, the $m^{2}$-neighborhood of $\kappa$ in $G$ contains $\tau$ (by $N_{m}(\iota(\kappa))=\chi$ and the definition of $m)$. Thus $\tau \backslash \kappa$ is contained in $N_{m^{2}}(\partial \kappa)$. Since $N_{m^{2}}(\partial \kappa)$ has $\leq d^{m^{2}}|\partial \kappa|=d^{m^{2}} n$ elements, $|\tau \backslash \kappa| \leq d^{m^{2}} n$. Now $G \backslash \kappa$ has only infinite connected components by minimality of the cutsets. Hence we can get $\kappa$ from $\tau$ by removing a subgraph $S$ of $\tau$ of size $\leq d^{m^{2}} n$ and such that any connected component of $S$ contains some element of $\partial_{i} \tau$. It thus suffices to show that there is an exponential bound on the number of such $S$ 's.

So let $\mathcal{S}$ be the set of subgraphs of $\tau$ of size $\leq d^{m^{2}} n$ with the property that each component contains a vertex of $\partial_{i} \tau$. Any element $S$ of $\mathcal{S}$ can be described as follows.

Let $S_{1}, \ldots, S_{k}$ be the components of $S$ and fix an element $r_{i}$ of $\partial_{i} \tau$ in $S_{i}$ for each $i$; let $R:=\left\{r_{1}, \ldots, r_{k}\right\}$. Now, if we first choose $R$ as a subset of $\partial_{i} \tau$, then choose the sizes of the $S_{i}$, and finally choose the actual subgraphs $S_{i}$ of $\tau$ of the given sizes and each incident to the corresponding element of $R$, then we obtain any possible $S \in \mathcal{S}$. One can choose $R$ as a subset of $\partial_{i} \tau$ (where $\left|\partial_{i} \tau\right| \leq d^{m^{2}} n$ ), in at most $2^{d^{m^{2}}{ }_{n}}$ ways. Once we have 
$\left\{r_{1}, \ldots, r_{k}\right\}=R$, we choose $\left|S_{i}\right|$ for each $i$ so that $\sum_{i=1}^{k}\left|S_{i}\right| \leq d^{m^{2}} n$ (by the definition of $\mathcal{S})$. This can be done in at most $\left(\begin{array}{c}d^{m^{2}} n \\ k\end{array}\right) \leq 2^{d^{m^{2}}{ }^{n}}$ ways. Finally, we choose the particular $S_{i}$ 's, knowing their sizes. By Lemma 2.1, there are at most $d^{2\left(\left|S_{1}\right|+\ldots+\left|S_{k}\right|\right)} \leq d^{2 d^{m^{2}}{ }^{n}}$ ways to do so.

We got that there are $\leq 2^{d^{m^{2}} n} \cdot 2^{d^{m^{2}} n} \cdot d^{2 d^{m^{2}} n}=\left(4 d^{2}\right)^{d^{m^{2}}{ }^{n}}$ ways to choose $S$, thus $|\mathcal{S}|$ is exponentially bounded. This finishes the proof.

\section{§3. The invariance of finiteness of $C_{G}$.}

In this section we show that if $C_{G}$ is infinite for a graph $G$, then $C_{G^{\prime}}$ is infinite for any graph $G^{\prime}$ that is bijectively quasi-isometric to $G$. This means that the answer to Question 2 of $[\mathrm{BB}]$ is positive.

Note that a minimal cutset $\Pi$ between two vertices $x$ and $y$ is also a minimal cutset between one of them and an end. Otherwise there are paths from $x$ and from $y$ to an end that do not intersect $\Pi$, and these could be used (by the definition of ends) to find a path between $x$ and $y$ that does not intersect $\Pi$, giving a contradiction. Hence the supremum in the definition of $C_{G}$ remains the same with the extra hypothesis that $y$ is in $G_{+} \backslash G$.

Consider a minimal cutset that separates a finite subgraph $X$ from infinity, and any boundary vertex $v$ of $X$. There is an infinite path starting from $v$ and going to infinity without intersecting $X$ in any point other than $v$, since any component of $G \backslash X$ is infinite by minimality.

Lemma 3.1. Suppose that a minimal cutset $S$ that separates the connected subgraph $X$ of $G$ from $\xi \in G_{+} \backslash G$ is not l-close. Then the set $S_{n}$ of edges that separate $N_{n}(X)$ from $\xi$ is a minimal cutset that is not $(l-2 n)$-close.

Proof. We may assume that $l \geq 2 n$. Let $A$ and $B$ partition $S$ so that $\operatorname{dist}(A, B)>l$. Then we have $N_{n}(A) \cap N_{n}(B)=\varnothing$. The set of vertices in $N_{n}(X)$ that are incident to some edge in $S_{n}$ is in $N_{n}(A) \cup N_{n}(B)$, and it has nonempty intersection with both $N_{n}(A)$ and $N_{n}(B)$ because of our condition about the paths. Hence the partition generated on $S_{n}$ by $N_{n}(A)$ and $N_{n}(B)$ shows that it is not $(l-2 n)$-close.

Theorem 3.2. Suppose that $C_{G}$ is infinite for the graph $G$. Then $C_{G^{\prime}}$ is infinite for any graph $G^{\prime}$ that is bijectively quasi-isometric to $G$.

Proof. Let $\iota: V(G) \rightarrow V\left(G^{\prime}\right)$ be a bijective quasi-isometry with constant $m$. For each $k$, let $G_{k}$ be a connected subgraph in $G$ whose boundary is not $k$-close, and such that from any point of $\partial G_{k}$ there is a path to infinity not intersecting $G_{k}$. Such subgraphs exist 
by our assumption on $G$ and the remark about the definition of $C_{G}$. As in the previous section, the $m$-neighborhood $N_{m}\left(\iota\left(G_{k}\right)\right)$ is connected. This implies that the set $S_{k}$ of edges that separate $N_{m}\left(\iota\left(G_{k}\right)\right)$ from infinity is a minimal cutset. By Lemma $3.1, S_{k}$ is not $(k / m-2 m)$-close. So the $S_{k}$ 's provide us with a sequence of minimal cutsets where the distances for certain partitions tend to infinity. This shows that $C_{G^{\prime}}$ is infinite.

REMARK. The assumption that $G$ and $G^{\prime}$ are bijectively quasi-isometric was not necessary in the last two sections. Basically similar arguments show that the conclusions remain true for any two quasi-isometric bounded degree graphs.

\section{$\S 4$. The lamplighter group has infinite $C_{G}$.}

In this section we answer Question 3 in [BB].

The lamplighter group is defined as the semidirect product of $\mathbb{Z}$ with $\sum_{x \in \mathbb{Z}} \mathbb{Z}_{2}$. For elements $p_{1}, p_{2} \in \mathbb{Z}$ and $l_{1}, l_{2} \in \sum_{x \in \mathbb{Z}} \mathbb{Z}_{2}$, the product is defined as

$$
\left(p_{1}, l_{1}\right)\left(p_{2}, l_{2}\right):=\left(p_{1}+p_{2}, l_{1} \oplus S^{-m_{1}} l_{2}\right)
$$

where $S$ is the left shift, $S(l)(i)=l(i+1)$ and $\oplus$ is componentwise addition mod 2. One can think of the elements of the lamplighter group as configurations where at each integer there is a lamp, either switched on or off, and there is a lamplighter standing at one of the integers. A possible set of generators is $\{(1, \omega),(0, \lambda)\}$, where $\omega$ stands for the sequence of all zeros, and $\lambda$ for the sequence of all zeros but a 1 in the 0 'th position. The first generator corresponds to that the lamplighter moves one step to the right, and the second one to that he switches the lamp in his current position.

THEOREM 4.1. If $G$ is a Cayley graph of the lamplighter group then $C_{G}$ is infinite.

The Diestel-Leader graph $D L(k, n)$ is constructed as follows. Let $T$ and $T^{\prime}$ be a $k+1$ regular and an $n+1$-regular tree respectively, and suppose that they are rooted at infinity so that their vertices are arranged into levels corresponding to the integers. Do it so that a vertex of $T$ on the $i$ 'th level has $k$ children on the $i+1$ 'th level and the parent on the $i-1$ 'th level; a vertex of $T^{\prime}$ on the $i$ 'th level has $n$ children on the $i-1$ 'th level and the parent on the $i+1^{\prime}$ th level. Let the level of $v$ in $T$ (resp. $T^{\prime}$ ) be denoted by $l_{T}(v)$ (resp. $\left.l_{T^{\prime}}(v)\right)$. $D L(k, n)$ is defined on the vertex set $\left\{\left(x, x^{\prime}\right) \in V(T) \times V\left(T^{\prime}\right): l_{T}(x)=l_{T^{\prime}}\left(x^{\prime}\right)\right\}$. There is an edge between $\left(x, x^{\prime}\right)$ and $\left(y, y^{\prime}\right)$ iff $x$ and $y$ are connected in $T$ and $x^{\prime}$ and $y^{\prime}$ are connected in $T^{\prime}$.

It is well known that $D L(2,2)$ is isomorphic to a Cayley graph of the lamplighter group. Briefly, fix a biinfinite path $R$ in $T$ and a biinfinite path $R^{\prime}$ in $T^{\prime}$ so that these 
paths intersect each level in exactly one vertex. For each vertex in $T$ and $T^{\prime}$, call the edge that goes to one of its children a 0-edge, and the other one a 1-edge. Do it so that the paths $R$ and $R^{\prime}$ contain only 0-edges. Now, the level of a vertex $\left(x, x^{\prime}\right)$ in $D L(2,2)$ is the position of the lamplighter, and what the lamplighter sees on his left (right), is just the sequence of 0 's and 1's on the edges of the infinite path connecting $x\left(x^{\prime}\right)$ to the root in infinity in $T\left(T^{\prime}\right)$, meaning the infinite path that always goes towards parents. For more details, see [Wo2].

Fix a vertex $o$ on the 0 'th level of $T$ and a vertex $o^{\prime}$ on the $k^{\prime}$ th level of $T^{\prime}$. Let the subtree $F_{k}\left(F_{k}^{\prime}\right)$ consist of the offspring of $o\left(o^{\prime}\right)$ of distance at most $k$ from it. Let $H_{k}$ be the subgraph $\left\{\left(x, x^{\prime}\right): x \in F_{k}, x^{\prime} \in F_{k}^{\prime}\right\}$ in $D L(2,2)$ and $C_{k}$ be the set of its boundary edges. Notice that $C_{k}$ is a minimal cutset and that it is the disjoint union of edges incident to $A_{k}=\left\{\left(x, x^{\prime}\right): \operatorname{deg}_{F_{k}}(x)=1\right\}$ and $B_{k}=\left\{\left(x, x^{\prime}\right): \operatorname{deg}_{F_{k}}\left(x^{\prime}\right)=1\right\}$ respectively. The distance of $A_{k}$ and $B_{k}$ is obviously $k$. Hence the sequence $C_{k}$ shows that $C_{D L(2,2)}$ is infinite.

\section{$\S 5 . C_{G}$ in finitely presented groups.}

The result in this section is the key in $[\mathrm{BB}]$ to proving $p_{u}<1$ for finitely presented groups with one end. We present a shorter proof here, using elementary arguments and terminology, as opposed to their argument using cohomology groups.

We use the obvious correspondence between subsets of $E(G)$ and the elements of $\{0,1\}^{E(G)}$ regarded as vectors, where $\bmod 2$ addition on these later corresponds to symmetric differences in the case of the subsets. Given a set $K$ of cycles in a graph $G$, we say that a cycle $C$ in $G$ is generated by $K$ if $C$ can be written as a mod 2 sum of cycles from $K$. Note that any cycle in a Cayley graph of a finitely presented group is generated by the set of cycles of length at most $t$, where $t$ is the maximal length of relators in this presentation of the group.

THEOREM 5.1. Let $G$ be a graph such that any cycle is generated by a set $K$ of cycles in $G$. Suppose that any cycle in $K$ has length at most $t$. Let $\Pi$ be a minimal cutset separating a vertex $x$ from $y \in V(G) \cup\{\infty\}$. Then for any nontrivial partition $\Pi_{1} \cup \Pi_{2}$ of $\Pi$ there are vertices $x_{i} \in \Pi_{i}(i=1,2)$ such that $\operatorname{dist}\left(x_{1}, x_{2}\right) \leq t / 2$. I.e., $C_{G} \leq t / 2$.

Proof. It is enough to show that there is a cycle in $K$ that intersects both $\Pi_{1}$ and $\Pi_{2}$. By minimality of the cutset $\Pi$, there are paths $P_{i}$ between $x$ and $y, i=1,2$, such that $P_{i}$ does not intersect $\Pi_{3-i}$. We may write $P_{1}-P_{2}$ as a $\bmod 2$ sum of cycles from $K$ : $P_{1}-P_{2}=\sum_{c \in K^{\prime}} c$, for a certain $K^{\prime} \subset K$. Let $K_{1}^{\prime}$ be the set of those cycles in $K^{\prime}$ that 
intersect $\Pi_{1}$, and $K_{2}^{\prime}:=K^{\prime} \backslash K_{1}^{\prime}$. Define

$$
\theta:=P_{1}-\sum_{c \in K_{1}^{\prime}} c=P_{2}+\sum_{c \in K_{2}^{\prime}} c .
$$

The right hand side is the sum of cycles and paths that do not intersect $\Pi_{1}$, hence $\theta$ does not contain any edge of $\Pi_{1}$. On the other hand, since the only odd degrees that $\theta$ has are in $x$ and $y$, these two have to be in the same connected component of $\theta$. Thus, there is a path from $x$ to $y$ that intersects $\Pi_{2}$. Since $P_{1}$ does not, we deduce that some cycle in $K_{1}^{\prime}$ does. This cycle intersects both $\Pi_{1}$ and $\Pi_{2}$.

\section{$\S 6$. Sizes of cutsets tend to infinity.}

By the growth of a Cayley graph we mean the function that takes in $n$ the size of a ball of radius $n$.

For a rooted tree $T$ and $x \in V(T)$ let the subtree $T_{x}$ be defined as the set of all descendants of $x$ (including $x$ itself) and the edges induced by them.

A lexicographically minimal spanning tree $T$ of a Cayley graph $G$ is a subtree rooted at the origin and defined in the following way. Fix a linear ordering of the generators of $G$ and their inverses. For any vertex $v$ of the Cayley graph choose the word representing $v$ that is lexicographically minimal among all such words. There is a path in $G$ that represents this word; define $T$ to be the union of all these paths (as $v$ ranges through every vertex). The graph we get is indeed a spanning tree. Moreover, it is subperiodic, that is, for any $x$ in $V(T)$ there is an embedding of $T_{x}$ into $T$ that maps $x$ to $o$. The growth rates of $T$ and $G$ are the same. These are straightforward corollaries of the definition of $T$; for more details about lexicographically minimal spanning trees, see, for example, [LP].

LEMMA 6.1. If a subperiodic tree $T$ has finitely many infinite rays then it has linear growth.

Proof. For $x \in V(G)$ denote by $F_{x}$ the union of the finite components of $T \backslash\{x\}$ not containing $o$. Define $S:=\left\{x \in V(T):\left|F_{x}\right|>\left|F_{y}\right|\right.$ for every $y$ where $\left.\operatorname{dist}(o, y)<\operatorname{dist}(o, x)\right\}$. By subperiodicity of $T$, for any $x \in V(T)$ there is an embedding $\phi$ of $T_{x}$ into $T$ such that $\phi(x)=o$. Fix $x \neq o$ and a corresponding $\phi$. If $z \in S \cap T_{x}$ then $\phi\left(F_{z}\right)$ can not be a subset of $F_{\phi(z)}$ by the definition of $S$. So $\phi\left(F_{z}\right)$ has the property that some of its vertices are mapped into vertices of an infinite ray starting from $\phi(z)$ and not intersecting $o$. This infinite ray contains no other vertices from $\phi\left(T_{x}\right)$ but those few from $\phi\left(F_{z}\right)$. So there are at most as many different " $F_{z}$ 's" as pairwise disjoint infinite rays, that is, $\left|S \cap T_{x}\right|$ is finite. This can hold for any $x \neq o$ iff $|S|$ is finite. Hence $\left|F_{x}\right|$ is bounded for every $x$, and $T$ has linear growth. 
TheOREM 6.2. In any Cayley-graph $G$ and for $n>0$, there are only finitely many cutsets of size $n$ separating a fixed vertex o from infinity, unless $G$ is a finite extension of $\mathbb{Z}$.

Proof. Let $S_{r}$ stand for the sphere of radius $r$ around $o ; B_{r}$ for the ball. By a cutset we always mean a cutset separating $o$ from infinity.

If $G$ has infinitely many ends then it is nonamenable and has an exponential bound for the number of minimal cutsets of size $n$ by Lemma 2.1, as shown in [BB]. So we may assume that $G$ has one end. Fix $n>0$. Choose a lexicographically minimal spanning tree $T$ in $G$. If $T$ has finitely many ends then the group grows linearly by Lemma 6.1 . Hence it is a finite extension of $\mathbb{Z}$. (For a proof of that groups of linear growth are finite extensions of $\mathbb{Z}$, see, for example, Corollary 3.18 in [Wo1].) If $T$ has infinitely many ends then there is a ball around $o$ such that any minimal cutset of size $n$ intersects it, namely a ball such that there are at least $n+1$ disjoint infinite rays starting from its boundary. Choose $X$ to be a set of edges that occurs in infinitely many minimal cutsets of size $n$ (to prove by contradiction), and maximal with this property. (So $0<|X|<n$.) Since $X$ is not a cutset and $G$ has one end, there are numbers $R$ and $r$ such that any vertex in $S_{r}$ is connected to $o$ by a path in $G \backslash X$ and not intersecting $S_{R}$. But then a cutset that has no edge in $B_{R}$ but those of $X$ cannot be minimal (it is necessarily a cutset without $X$ too). This shows, by the choice of $X$, that there cannot be infinitely many minimal cutsets of size $n$ and containing $X$. This contradiction finishes the proof.

Acknowledgements. I thank Russell Lyons and Gábor Pete for their comments on the manuscript.

\section{REFERENCES}

[BB] E. Babson and I. Benjamini. Cut sets and normed cohomology with applications to percolation. Proc. Amer. Math. Soc., 127:589-597, 1999.

[BS] I. Benjamini and O. Schramm. Percolation beyond $\mathbb{Z}^{d}$. Electr. Commun. Prob., 1:7182, 1996.

[CS] T. Coulhon and L. Saloff-Coste. Isopérimétrie sur les groupes et les variétés. Rev. Mat. Iberoamericana, 9:293-314, 1993.

[dlH] P. de la Harpe. Topics in Geometric Group Theory. Chicago Lectures in Mathematics Series, 2000.

[Ke] H. Kesten. Percolation Theory for Mathematicians. Birkhäuser, Boston.

[LP] R. Lyons and Y. Peres, Probability on Trees and Networks, in preparation. http://mypage.iu.edu/ rdlyons/prbtree/prbtree.htm 
[Wo1] W. Woess. Random Walks on Infinite Graphs and Groups. Cambridge Tracts in Mathematics, Vol. 138, 2000.

[Wo2] W. Woess. Lamplighters, Diestel-Leader graphs, random walks, and harmonic functions. Combinatorics, Probability and Computing, to appear.

Department of Mathematics, Indiana University, Bloomington, IN 47405-5701

atimar@indiana.edu 